\title{
Seismocardiography on Infants and Kids
}

\author{
Nico Jähne-Raden ${ }^{1}$, Henrike Gütschleg ${ }^{1}$, Marie Cathrine Wolf ${ }^{1},{\text { Stephan } \operatorname{Sigg}^{2}, \text { Ulf Kulau }}^{3}$ \\ ${ }^{1}$ Peter L. Reichertz Institute - Hannover Medical School, Hanover, Germany \\ ${ }^{2}$ Dept. of Communications and Networking - Aalto University, Aalto, Finland \\ ${ }^{3}$ DSI Aerospace, Bremen, Germany
}

\begin{abstract}
Seismocardiography (SCG) offers a variety of possibilities for cardiovascular diagnostics, especially in long-term monitoring. Unfortunately, little information is available about changes in SCG-signal-morphology over the life course, as well as differences in different age groups. A highly specialized system with two isochronous accelerometers (at sternum/apex) and a reference ECG with a sample frequency of $17 \mathrm{kHz}$ was used. We included healthy infants and children between 0 and 14 years, with a focus on babies. In contrast to adult measurements, working with children is demanding in many ways (e.g. movement artifacts). First, the SCG and ECG data were processed by filter mechanisms and annotated by peakdetection based on patter analysis. With the annotated data, a detailed examination of the established SCG features with regard to absolute and relative amplitudes and time intervals was realized. Through this analysis it is possible to make initial comparisons of the child groups to adult data. The infant's SCG amplitude is up to five times smaller, but the actual signal morphology is equal. Established physiological processes of the child's heart can also be identified (e.g. high pulse-rate-variability). This work is a prelude to a further collection and should lead to an open-data database that includes data a wide range of ages as well as various pathologies.
\end{abstract}

\section{Introduction}

Seismocardiography (SCG) offers a variety of possibilities for cardiovascular diagnostics, especially in long-term monitoring. With this measurement of SCG signals on people with healthy heart, using only highly sensitive, digital, commercially available, tri-axial acceleration sensors, a baseline can be recorded. By integrating different measuring points and the possibility of highly synchronous measurement including an ECG reference signal, the relationship of SCG signals from different measuring positions is also examined. With the focus on children and especially infants, physiological changes can be further investigated.

Physiologically, the change in the human body and notably in the heart has been extensively investigated with increasing age (e.g. doubled oxygen demand, heart size ratios differs). From birth to adolescence, the resting heart rate drops (about twice as high in new-borns) and the ejection volume increases as the body grows. This work is intended to provide a descriptive representation of changes in the SCG signal using an optimized measurement system. At the present time only a few SCG studies involving children have been carried out (cf. [1,2]).

Hence, little is known about the SCG signal morphology in children or infants. Certainly, physiological differences in the heart of children, in contrast to adults, can be recorded very quickly, e.g. the higher beating rate of the heart or the higher rate variability. The measurement of SCG signals by means of a high-precision measuring system can serve as a further component for understanding the SCG basics as well as pathological changes.

The overall goal of the data recording of the feasibility study is to determine the usability of the created SCG measurement system, consisting of body-worn acceleration sensors and a computer unit, for use on children. As a secondary goal, these series of measurements are intended to examine and design the possibility of improving the created system for this very purpose, and to generate the first children's signals for expanding the existing SCG data portfolio.

\section{Method}

The technical structure of the overall FieldProgrammable-Gate-Array (FPGA) system described in section [3] was used for recording the SCG signals (Zedboard Evaluation Board incl. Xilinx Zynq-7020 System on Chip dual-core ARM processor with a Virtex7FPGA). Three accelerometers (KX1022-1037 from Kionix) with a setting of 16,384 counts/g at $2 \mathrm{~g}$ were then used via SPI. In addition, a proprietary ECG measuring system (OLIMEX ECG SHIELD) using a 1-channel LARA derivation as reference, connected via a 10-bit 
ADC. All SPI controllers use a common clock line so that reading out the data is absolutely synchronous. Since the parallel SPI controllers are implemented in hardware, a high data rate can be achieved. One axis of several accelerometers can be read out simultaneously with a data rate of up to $20 \mathrm{kHz}$. Each sample is provided with a time stamp in hardware and forwarded to an SRAM buffer. The buffer can be accessed via Xillybus, which enables a Direct-Memory-Access connection from the ARM cores to the FPGA. An embedded Linux is executed on the ARM cores, which enables data processing, forwarding and storage. To improve the signal quality, an internal sampling frequency of $800 \mathrm{~Hz}$ was selected for the accelerometer. In addition, each sensor performs a lossfree, internal averaging of 128 samples. Due to the significantly higher working frequency of the Zedboards, an eleven-fold oversampling is generated for each accelerometer axis. With the setting used, a stable 17,400 samples per second can be achieved for each accelerometer axis. For the ECG signal, 12,800 samples per second for the three analogue channels are achieved through the more complex SPI control. The measuring system allows high data quality (low signal-to-noise behaviour), high signal resolution ( $2 \mathrm{~g}$ at $16 \mathrm{bit})$. In addition, clock-synchronous readout of the two accelerometers channel at a suitable sample frequency, aimed at a two-digit kilohertz range including oversampling, this ensures data robustness. A time shift of the recording of about $20 \mathrm{~ns}$ provides a realtime measurement of the SCG parameters.

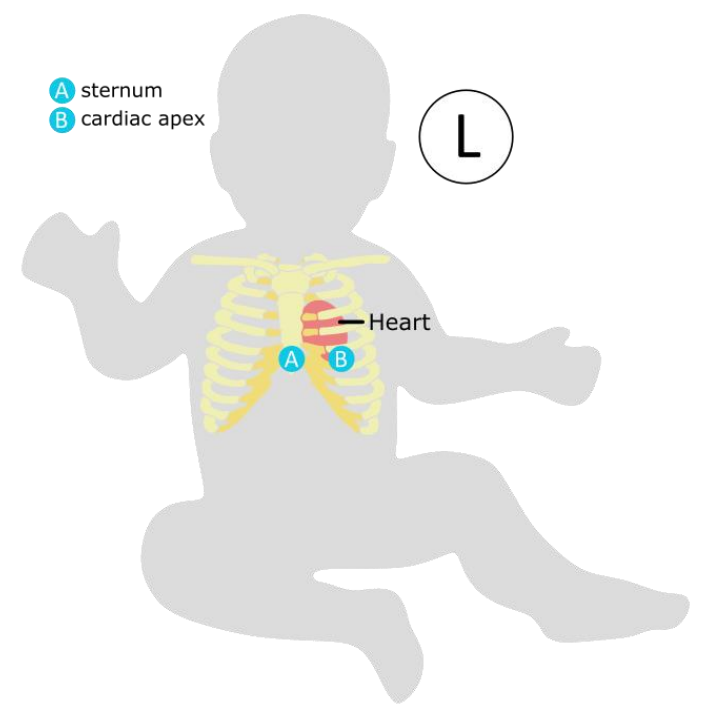

Figure 1. Model of the two measurement positions.

The measurement positions were limited to two measurement points, from overall six in the adult subject measurements. This also relieved the children, as the time required to prepare for measurements could be reduced. For the reference measurement, an 1-channel ECG was read out via the analogue output parallel to the acceleration signals. The measurement positions are both on the thorax, on the one hand on the sternum (see figure 1 position A) and on the other hand at the cardiac apex position, medioclavicular at the level of the fifth intercostal space (position B).

During the measurement, the child test subjects are in a resting position, preferring lying down, although the focus is on comfort and compliance, especially for the youngest test subjects. The measurement itself was limited to at least two minutes to about three minutes as a maximum. Attempts were made to avoid discussions and overly hectic movements by the test subjects during the measurement, among other things through the constant presence of the respective parents.

Due to the exploratory nature of this study, a significant number of cases was not estimated. A total of twelve subjects in five classes ( $0-1$ year,> 1-3 years,> 3-6 years,> 6-10 years,> 10-14 years) were included. The greatest interest in the survey was in the youngest group, since the greatest difference in signal morphology to adults was expected here. This resulted in a division of 4-2-2-2-2 for this series of measurements. In the figure 2 the upper bodies of two subjects can be seen on the one hand from the group under the age of one and on the other hand from the group between the ages of ten and fourteen years.

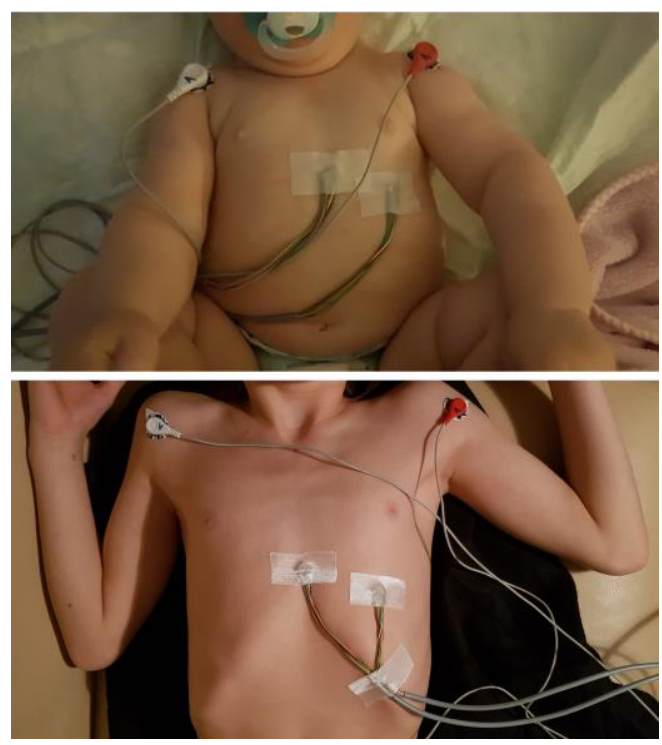

Figure 2. Fotos of the upper bodies of two subjects (infant on top and child between 10-14y on bottom).

For the series of measurements, the subjects' general heart health and age up to their fifteenth birthday were set as inclusion criteria, since the differences in physiology among adults decrease with increasing age. Since the focus was on infants and kids, different age groups were formed. Known infectious diseases (e.g. MRSA), wounds that prevent measurement, severe overweight (from level I obesity, corresponding to a body mass index of $30 \mathrm{~kg} / \mathrm{m}^{2}$ ) 
and operations or ongoing therapies that influence the measurement were used as exclusion criteria. The basic classification with regard to suitability for the measurement was made through the visual assessment, as well as according to the statements of the participating children or their legal guardians, which are supported by the close-knit German health system, in particular the preventive medical examinations for children.

\section{Results}

The aim of the investigation was to determine structural differences (amplitudes, changes over time) in the signal morphology in relation to the SCG signals of heart-healthy adults. From this it is potentially possible to draw conclusions about unfavorable health changes within the signal in the event of a disease.

Table 1. Aggregated values for the amplitude, width and distance to the MC-peak, of the chosen signal parameters.

\begin{tabular}{|c|c|c|c|c|}
\hline & & amplitude [g] & width [s] & dist. to $\mathrm{MC}[\mathrm{s}]$ \\
\hline \multirow{6}{*}{ MC } & mean & 0,048168833 & 0,022836071 & 0 \\
\hline & median & 0,037144573 & 0,022017595 & 0 \\
\hline & sd & 0,038733646 & 0,00600424 & 0 \\
\hline & var & 43,29497506 & 0,072749218 & 0 \\
\hline & $\min$ & 0,013132172 & 0,014114462 & 0 \\
\hline & $\max$ & 0,270317854 & 0,046258701 & 0 \\
\hline \multirow{6}{*}{ IVC } & mean & 0,002966487 & 0,017242235 & 0,011880013 \\
\hline & median & 0,004667678 & 0,016386311 & 0,011069219 \\
\hline & sd & 0,033497097 & 0,0052879 & 0,003742966 \\
\hline & var & 27,95691698 & 0,051365668 & 0,026689057 \\
\hline & $\min$ & $-0,135264135$ & 0,008265661 & 0,007540603 \\
\hline & $\max$ & 0,166878082 & 0,03591454 & 0,026682135 \\
\hline \multirow{6}{*}{ AO } & mean & 0,015213691 & 0,011968799 & 0,017822281 \\
\hline & median & 0,010555303 & 0,011504254 & 0,016966357 \\
\hline & sd & 0,030231484 & 0,004767099 & 0,0052879 \\
\hline & var & 24,19260191 & 0,039810507 & 0,051365668 \\
\hline & $\min$ & $-0,10550869$ & 0,002561872 & 0,008845708 \\
\hline & $\max$ & 0,169843113 & 0,027068832 & 0,036494586 \\
\hline \multirow{6}{*}{ RE } & mean & 0,021056876 & 0,013113396 & 0,037087657 \\
\hline & median & 0,015630339 & 0,012325986 & 0,037171307 \\
\hline & sd & 0,029914992 & 0,00475487 & 0,009658356 \\
\hline & var & 24,00626047 & 0,040665915 & 0,163341464 \\
\hline & $\min$ & $-0,082033505$ & 0,00362529 & 0,01537123 \\
\hline & $\max$ & 0,184599766 & 0,030549111 & 0,057859629 \\
\hline \multirow{6}{*}{$\mathbf{R F}$} & mean & 0,0174959 & 0,013454169 & 0,407847994 \\
\hline & median & 0,013188146 & 0,012470998 & 0,400618716 \\
\hline & sd & 0,024911507 & 0,005569479 & 0,028999931 \\
\hline & var & 17,70554537 & 0,056549412 & 1,511928847 \\
\hline & $\min$ & $-0,083838581$ & 0,003576953 & 0,369441222 \\
\hline & $\max$ & 0,144452663 & 0,036204563 & 0,549158933 \\
\hline
\end{tabular}

For the analysis of the signal data, a suitable bandpass filtering (Butterworth 3rd order $[0.25 \mathrm{~Hz}, 85 \mathrm{~Hz}]$ ) as well as a calibration of the data to the acceleration due to gravity (using a 3D ellipsoid, see [4]) was carried out. This was followed by the annotation of the individual heartbeats or the areas of the heartbeat (systolic, diastolic) as well as the established SCG signal characteristics (cf. [5, 6]). The anchor points of the annotation for the SCG data of the children and infants were chosen for the systolic signal range $\mathrm{MO}$ and the diastolic range RF. The table 1 shows the aggregated analysis results of the child measurement for all established systolic characteristics (MC, IVC, AO, $\mathrm{RE})$ and for the diastolic SCG signal characteristic of rapid filling (RF) (mean, median, standard deviation (sd), variance (var), minimum (min), maximum (max)).

In order to be able to take a more detailed look at the results of the subjects aged 0-2 years, the determined data were considered separately. Table 2 shows the comparative results for the MC-peak as an example. The younger subjects tend to have higher amplitudes of the MC. This also applies to IVC and RE. In the diastolic area, only the RF was initially found, with a slightly increased amplitude compared to adults. The standard deviation and variance are also significantly increased in the signal data of the younger test subjects. The extreme values, however, come about through additional unavoidable movements of the test persons. The representation of the minimum values shows that this cannot be transferred to all aggregated values. The color representation in Table 2 marks the higher values in red and the lower values in green. The color intensity depends on the value.

Table 2. Aggregated values for the amplitude, width and distance to the MC-peak, of the chosen signal parameters.

\begin{tabular}{|c|c|c|c|c|c|}
\hline age & mean & sd & var & $\max$ & $\min$ \\
\hline \multirow{4}{*}{$<1 y$} & 0,05499 & 0,07604 & 94,4292 & 0,58787 & 0,01245 \\
\hline & 0,06082 & 0,08973 & 131,3688 & 0,56280 & 0,01313 \\
\hline & 0,05084 & 0,03697 & 22,3128 & 0,51044 & 0,01317 \\
\hline & 0,09429 & 0,10293 & 172,9190 & 0,58141 & 0,01226 \\
\hline \multirow{2}{*}{$<2 y$} & 0,05278 & 0,04550 & 33,7453 & 0,29549 & 0,01306 \\
\hline & 0,07825 & 0,05821 & 55,1759 & 0,32587 & 0,01380 \\
\hline \multirow{6}{*}{$>2 y$} & 0,03020 & 0,00749 & 0,9167 & 0,05132 & 0,01243 \\
\hline & 0,04127 & 0,01461 & 3,4814 & 0,07597 & 0,01334 \\
\hline & 0,02092 & 0,00456 & 96 & 0 , & 251 \\
\hline & 0,03782 & 0,01183 & 2,2821 & 0,06626 & 0,01448 \\
\hline & 0,02166 & 0,00575 & 0,5376 & 0,04433 & 0,01236 \\
\hline & 0,03417 & 0,01117 & 2,0313 & 0,10304 & 0,01460 \\
\hline
\end{tabular}

Figure 3 shows an example of the signal characteristics of a child.

The aggregated properties are shown in box plots for the amplitudes (vertical) and the distances (horizontal). In the upper area, the standard plot of a signal characteristic as well as the middle signal course is shown. 


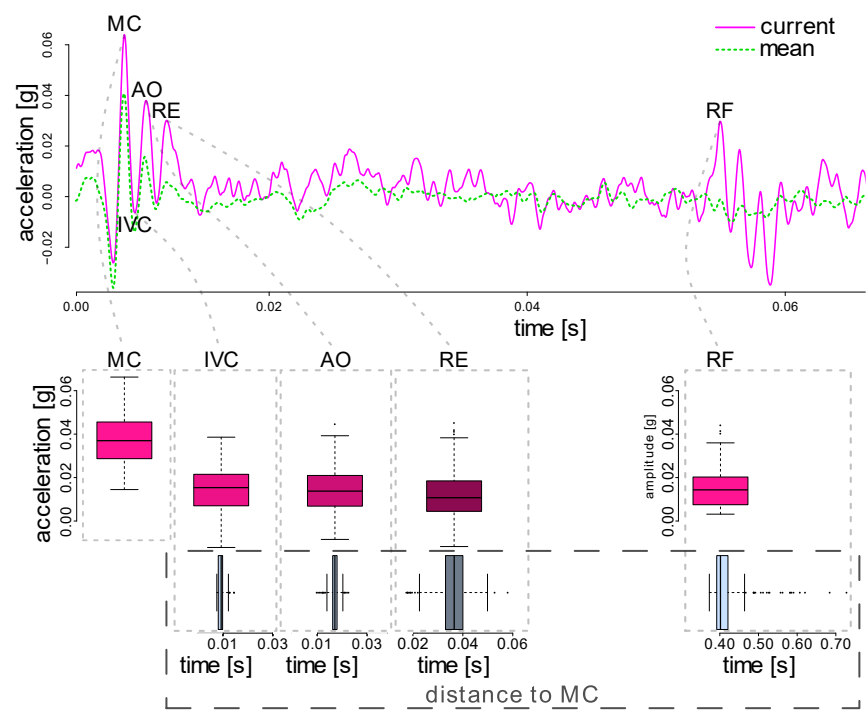

Figure 3. Summary plot of one subject with aggregated box plots for some significant signal parameters.

\section{Conclusion}

When examining the feature amplitudes, the structure of Zanetti et al. (see [5, 6]) confirmed. It was shown that the structure of the children's data is similar to that of adults. The amplitudes of the MO feature are also comparable, although the other amplitudes can also differ greatly.

Looking at these aggregated results, there are no noticeable deviations in the amplitude level compared to the adult, heart-healthy volunteers who were included in a comparable study (see [3]).

When looking at the time parameters, a plausible shortening becomes apparent due to the physiologically normal, age-related increased pulse rate of the child test subjects. In addition, it was possible to determine a significantly increased variance in the time courses compared to the adult test subjects. The pulse rate of infants and children changes more quickly, as is known for this age group.

However, this also depends on the measuring position and can possibly be caused by a displacement of this. The results of the 0-2 years old test persons were examined in a special way. Here, however, there are clear deviations in the amplitude of the child subjects. The amplitude is on average about 2-5 times that of a healthy adult. This possibly relates to the relatively large mass ratio of heart and body in infants. Table 2 shows a clear change in the sd, the var and the max amplitude values. Although attempts were made to clean up the data from movement artifacts of the children as best as possible, it cannot be ruled out that these have an influence.

In addition, the SCG signals of heart-healthy infants and children were recorded with an adapted measurement technique and setup in order to determine if necessary physiological changes in the growth process and, if necessary, to detect a pathological course. These extensive studies have led to the determination of direct HardwareSoftware properties, as well as the subsequent procedural requirements of a measurement setup or implementation. This knowledge and the associated information led to the implementation of a final research prototype for the measurement of BCG and SCG signals from heart failure subjects.

\section{Acknowledgments}

The implementation of this study was checked in advance by an independent ethics committee and classified as harmless (vote: 13.12.2019, No. 8758\_BO \SS _2019, ethics committee of the Hannover Medical School, Hanover, Germany, on the study: "Sensorbasierte ballistokardiografische Messung bei Kindern").

\section{References}

[1] A. Taebi, B. Solar, A. Bomar, R. Sandler, "Recent Advances in Seismocardiography" Vibrations, vol. 2, no. 1, pp. 64-86, 2019.

[2] N. Jähne-Raden, H. Gütschleg, M. Marschollek, "Trodden Lanes or New Paths: Ballisto- and Seismocardiography Till Now", Studies in Health Technology and Informatics, vol. 270, pp. 479-483, 2020.

[3] N. Jähne-Raden, H. Gütschleg, T. Clausen, T. Jura, U. Kulau, S. Sigg, "High-Resolution Synchronous Digital Ballistocardiography Setup", 2019 Computing in Cardiology Conference (CinC), Computing in Cardiology Conference (CinC), vol. 46, Sep. 2020.

[4] M. Gietzelt, K-H. Wolf, M. Marschollek, R. Haux, "Performance Comparison of Accelerometer Calibration Algorithms based on \{3D\}-Ellipsoid Fitting Methods", Comput Methods Programs Biomed, vol. 111, no. 1, pp. 6271, Sep. 2013.

[5] J. Zanetti, K. Tavakolian, "Seismocardiography: Past, Present and Future", Conference proceedings Annual International Conference of the IEEE Engineering in Medicine and Biology Society, IEEE Engineering in Medicine and Biology Society, vol. 2013, pp. 7004-7007, 2013.

[6] O. Inan, P. Migeotte, K. Park, M. Etemadi, K. Tavakolian, R. Casanella, J. Zanetti, J. Tank, I. Funtova, K. Prisk, M. Di Rienzo, "Ballistocardiography and Seismocardiography: A Review of Recent Advances", IEEE Journal of Biomedical and Health Informatics, vol. 19, no. 4, pp. 1414-1427, 2015.

Address for correspondence:

Nico Jähne-Raden

Peter L. Reichertz Institute for Medical Informatics of TU Braunschweig and Hannover Medical School, Hannover Medical School, Carl-Neuberg-Str. 1, D-30625 Hanover, Germany. Nico.Jaehne-Raden@plri.de 